\title{
Experimental Studies on Permeability of Tracers into the Spinal Cord
}

\author{
S. Kashiwaguchi, MD, K. Masaki, MD, T. Ikata, MD \\ Departments of Orthopedic Surgery, School of Medicine, Tokushima University, \\ Japan.
}

\section{Summary}

For investigation of the intramedullary penetration of metrizamide, the intramedullary movements of fluorescein, Evans Blue, Horseradish peroxidase (HRP) and ionic lanthanum in the normal cord and experimental syringomyelia of rats were studied. Syringomyelia was induced by injection of kaolin suspension into the cisterna magna. Subsequently HRP or ionic lanthanum was perfused into the subarachnoid space and its distribution was examined by electron microscopy.

In normal rats, HRP mainly became localised in the basement membrane between the pia mater and glial cells and only a few vesicles migrated into the extracellular spaces (ECS) between glial cells. Ionic lanthanum, however, migrated deep into the ECS. In rats with experimental syringomyelia many vesicles of HRP migrated deep into the ECS of glial cells and the white matter.

These findings indicated that the ECS of the marginal glia and the neuropil constitute part of a pathway through which metrizamide migrates into the intramedullary cavity.

Key words: Subarachnoid perfusion; Intramedullary penetration; Syringomyelia; Marginal glia.

Metrizamide computed tomography followed by delayed scanning (DCTM) is useful clinically in the diagnosis of intramedullary lesions of the spinal cord, especially syringomyelia. The way in which intrathecally injected metrizamide migrates into the cavity is still unknown, although reflux into the central canal of the cord via the obex has been postulated. In our 18 patients whose syringomyelia was examined by DCTM, the attenuation values within the parenchyma increased progressively although those of the subarachnoid space decreased. These findings suggest active metrizamide filling of the parenchyma and the cavity across the marginal glia (cerebrospinal fluid-spinal cord barrier). Since penetration of metrizamide into the normal brain has been demonstrated by CT and autoradiography (Golman, 1973; Drayer and Rosenbaum, 1977), similar penetration into the spinal cord might be expected. Intramedullary pene- 
Table Materials

\begin{tabular}{lr}
\hline Wistar rats & $\mathrm{n}=26$ \\
\hline Untreated rats & $\mathrm{n}=16$ \\
Fluorescein & \\
Evans Blue & 6 \\
HRP & 5 \\
Ionic lanthanum & 5 \\
Experimental syringomyelia rats & \\
HRP & $\mathrm{n}=10$ \\
\hline
\end{tabular}

tration of metrizamide in normal adult dogs has been shown by DCTM (Dubois, 1981), and increased permeability has been observed in dogs with experimental syringomyelia (Henmi, 1985). In this study, the intramedullary penetration of tracers was investigated after their subarachnoid perfusion in normal cord and experimental syringomyelia of rats.

\section{Materials and methods}

The intramedullary penetration of tracers was studied in 16 normal rats and 10 rats with experimental syringomyelia (Table).

\section{Control group}

Sixteen untreated rats were used as controls. The rats were anesthetised by i.p. injection of pentobarbital (Nembutal $0.1 \mathrm{ml}$ per $100 \mathrm{~g}, 60 \mathrm{mg} / \mathrm{ml}$ ), and an inflow catheter $(\varphi 0.05 \mathrm{~mm})$ was introduced into the subarachnoid space at the lumbar level (L6) by a microsurgical technique. An outflow slit was made in the exposed dura mater at the cisterna magna. The subarachnoid space was then perfused from the lumbar level to the cisterna magna. Three studies on tracers were carried out as follows:

Evans Blue and fluorescein. A solution of Evans Blue $(2 \%)$ and fluorescein $(2 \%)$ in saline was perfused into the subarachnoid space at $0.2 \mathrm{ml}$ per hour for 1 hour (Fig. 1A, Fig. 1B). The spinal cord was fixed by the transcardiac route with a solution of $10 \%$ formaldehyde by an infusion drip. Then the spinal cord was promptly removed and coronal sections of approximately 1-2 $\mathrm{mm}$ thickness were examined in a stereoscopic microscope.

HRP. Horseradish peroxidase (HRP; MW 43,000; Sigma type II, $10 \mathrm{mg} / \mathrm{ml}$ ) dissolved in saline was perfused into the subarachnoid space for 2 hours. The spinal cord was fixed by the transcardiac route with a solution of $4 \%$ paraformaldehyde and $0.5 \%$ glutaraldehyde in $0.1 \mathrm{M}$ phosphate buffer, $\mathrm{pH} 7 \cdot 4$, by a similar perfusion procedure. The spinal cord was then promptly removed and placed in the same fixative for an additional 3 hours. Some parts were sectioned with a microslicer (MICRO SLICER DTK-1000) at a thickness of 50-100 $\mu \mathrm{m}$. The sections were incubated for peroxidase activity by means of a two step method (Westergarrd, et al.,). The portions of white matter conţaining pia mater in which dark HRP products were seen were cut out. The tissue blocks were postfixed in a buffered solution of $1 \% \mathrm{OsO}_{4}$ for 2 hours, and then dehydrated 

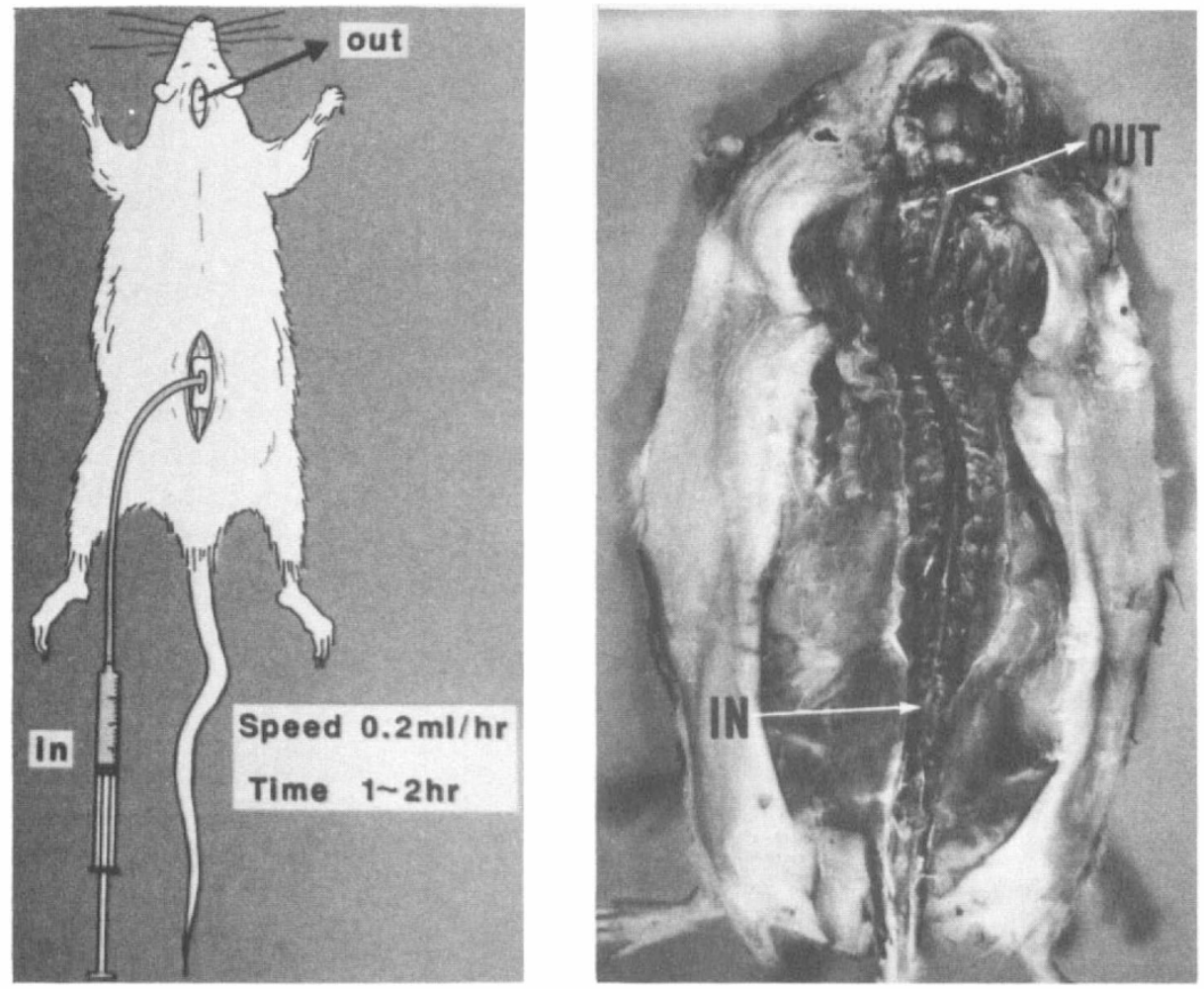

Figure 1A An inflow catheter was introduced into the subarachnoid space at lumbar level, and an outflow slit was made in the exposed dura mater at the cisterna magna. B The subarachnoid space was then perfused from lumbar level to the cisterna magna.

in a graded series of acetone and embedded in Epon. Ultrathin sections were examined in a Hitachi HU12 electron microscope with or without uranyl acetate and lead citrate staining.

Lanthanum. Lanthanum chloride $\left(\mathrm{LaCl}_{3} \mathrm{MW}\right.$ 138.9; Sigma, $\left.5 \mathrm{mM}\right)$ dissolved in saline was perfused into the subarachnoid space. The spinal cord was fixed by the transcardiac route with a solution of $4 \%$ paraformaldehyde and $0.5 \%$ glutaraldehyde in $0 \cdot 1 \mathrm{M}$ sodium cacodylate buffer at $\mathrm{pH} 7.4$ by a similar perfusion procedure to that described above. The spinal cord was then removed and placed in the same fixative for 3 hours. The portions dissected and examined were same as those in studies with HRP.

\section{Rats with experimental syringomyelia}

For induction of syringomyelia, 10 anesthetised Wistar rats, weighing about $200 \mathrm{~g}$, were injected $0.04-0.05 \mathrm{ml}$ of $25 \%$ kaolin suspension into the exposed cisterna magna. An inflow catheter was introduced into the subarachnoid space from the lumbar level 6 to 8 weeks later. The dura mater in the cisterna magna could not be cut, because of marked adhesion due to inflammation after kaolin injection. HRP $(10 \mathrm{mg} / \mathrm{ml})$ dissolved in saline was infused into the subarachnoid space at $0.2 \mathrm{ml}$ per hour for 2 hours. The spinal cord was fixed by the trans- 

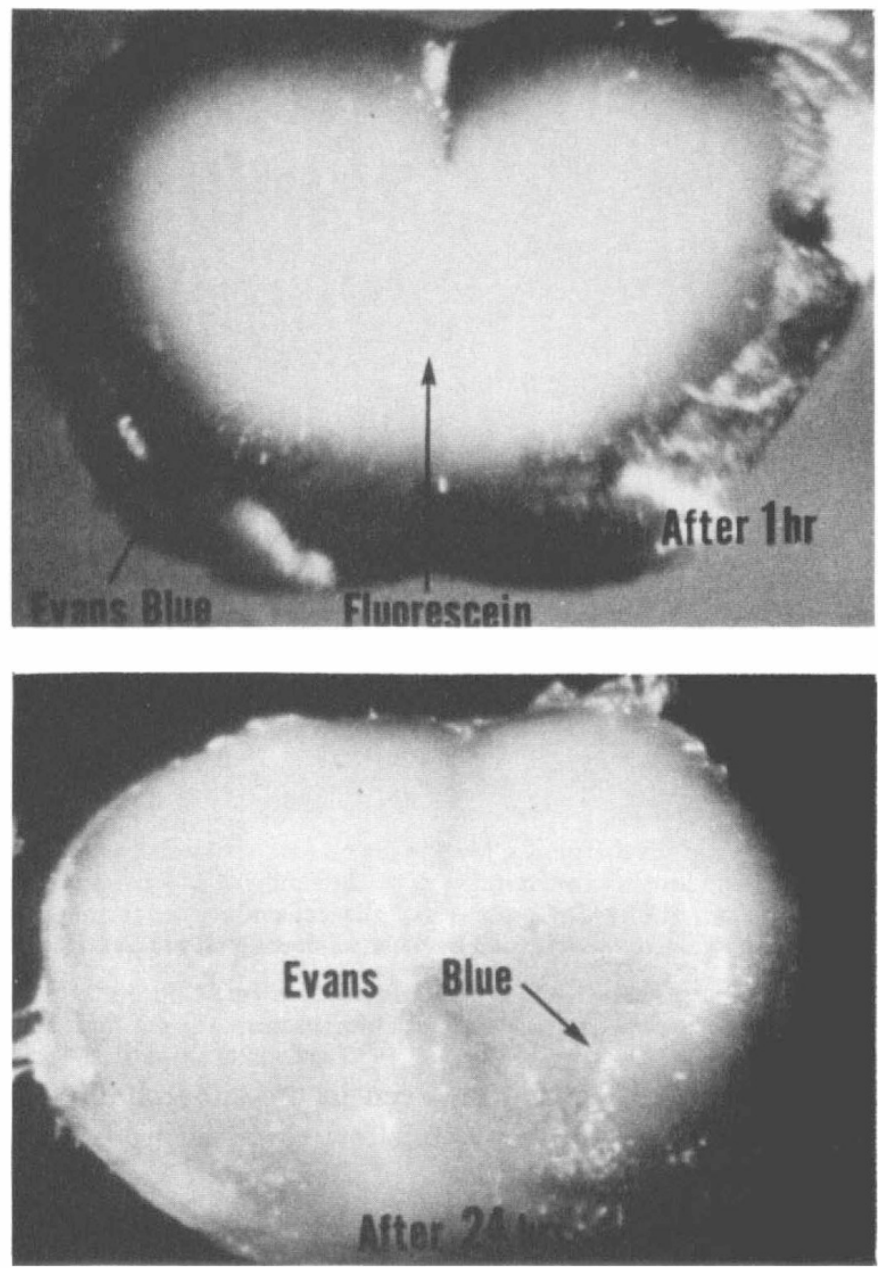

Figure 2A Perfusion of Evans Blue and fluorescein into the subarachnoid space. After perfusion for 1 hour, fluorescein was seen in the whole parenchymal area. B The specimen was taken out at 24 hours after the perfusion existed for 1 hour. Fluorescein was absent, but Evans Blue was vaguely present in the whole parenchymal area.

cardiac route and removed as described for the control group. The regions dissected and examined were same as in the control group.

\section{Results}

\section{Control group}

Evans Blue and fluorescein. After perfusion for 1 hour, fluorescein (MW 376) was seen in the whole parenchymal area, but Evans Blue (MW 789) was only in the peripheral region of the white matter. In the specimen which taken out at 24 hours after the perfusion existed for 1 hour, no fluorescein was seen, but Evans Blue was seen vaguely in the whole parenchymal area (Fig. 2A, Fig. 2B). 


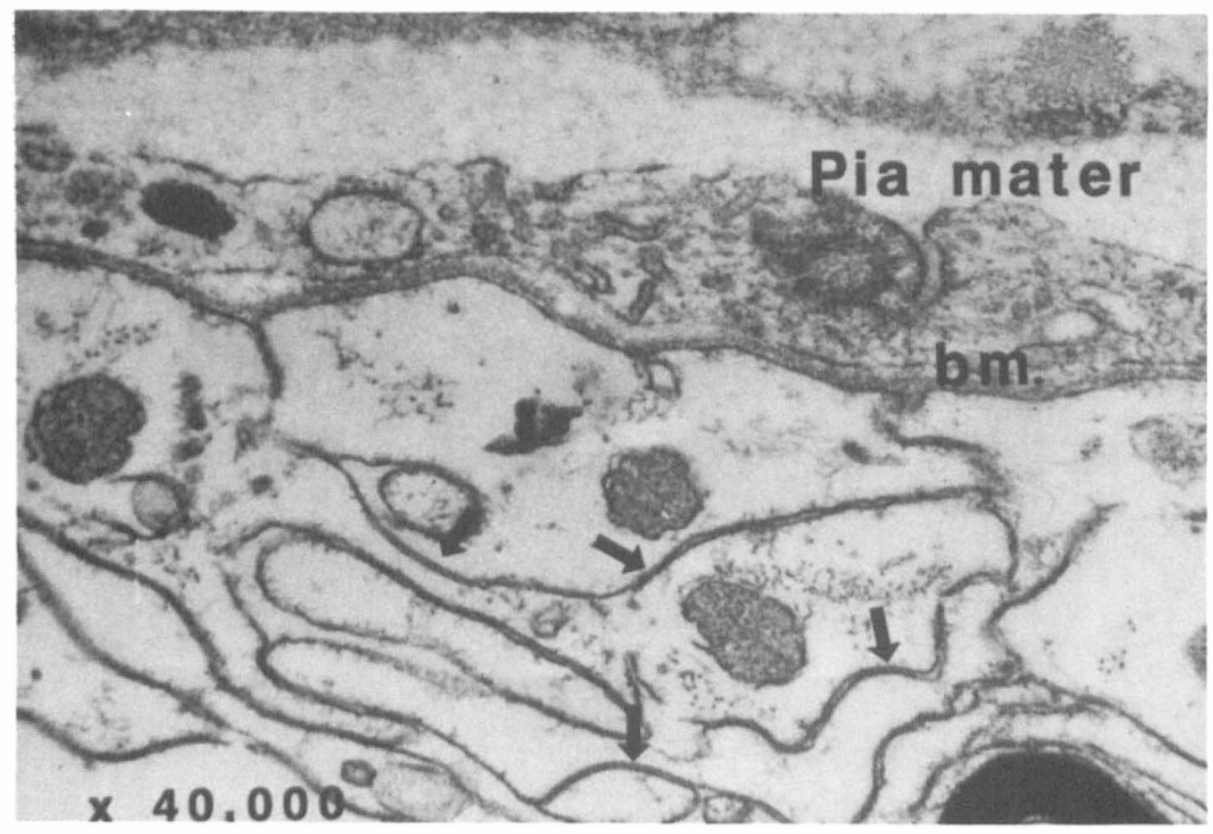

Figure 3 The pia mater and the marginal glia. The pia mater consisted of a network of flattened cells with long processes and interspersed bundles of collagen fibrils. The basement membrane covered the spinal parenchyma. The long processes of glial cells interlaced forming 'the marginal glia'. Numerous gap junction (arrow) were seen between adjacent glial processes.

HRP. Reaction products of HRP were seen in the subarchnoid space and the pia mater by stereoscopic microscopy, but few reaction products were seen in the parenchyma.

The microstructure of the surface of the spinal cord was examined by electron microscopy. The pia mater closely surrounding the spinal parenchyma consisted of a network of flattened cells with long processes and interspersed bundles of collagen fibrils. The long thin processes of the pial cells extended in various directions to contact neighbouring pial cells, but no junctions were identified in this layer. These processes were contacted in some places, and the basement membrane covered the spinal parenchyma. The long processes of the glial cells interlaced forming 'the marginal glia.' Numerous gap junctions were seen between adjacent glial processes (Fig. 3).

By electron microscopy, many dark precipitates of reaction products of HRP were seen in the subarachnoid space, the pia mater, the subpial space and the basement membrane of glial cells. A few products were also seen in the extracellular space (ECS) between the glial cells. However, only a few products were seen in the ECS around the neuropil (Fig. 4).

Lanthanum. The distribution of lanthanum in the pia mater and the basement membrane was similar to that seen with HRP. Many deposits of lanthanum were also observed in the ECS between the glial cells and around the neuropil (Fig. 5). However, no deposits of lanthanum were observed in the ECS of the grey matter. 


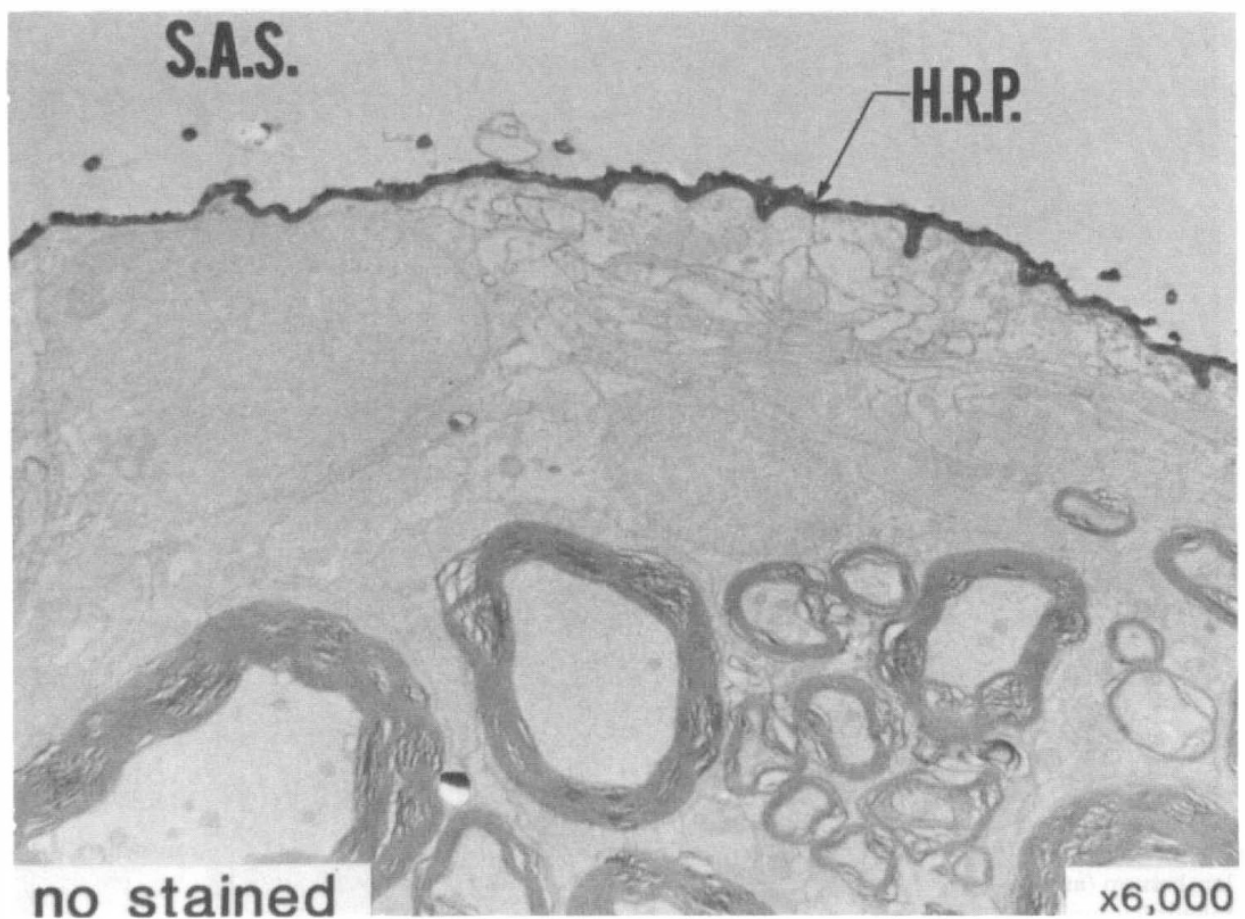

Figure 4 Normal adult rat. Many reaction products of HRP were seen in the subarachnoid space, the pia mater and the basement membrane. A few products were also seen in the extracellular space between the glial cells.

\section{Rats with experimental syringomyelia}

All rats showed slight to moderate syringomyelia (Fig. 6). Fluorescein and Evans Blue were seen in whole parenchymal area. The distribution of reaction products of HRP, however, was different from that in the control group: many products of HRP were seen not only in the ECS of the marginal glia but also in the ECS around the neuropil (Fig. 7, Fig. 8).

\section{Discussion}

Initially late migration of intrathecally injected metrizamide into syringomyelic cavities was proposed to indicate a communication between the intramedullary cavity and the fourth ventricle (Vignaud). However, this idea did not explain why increase in intracavitary opacity persisted after 'obex plugging', and so another route, 'intramedullary penetrance' was suggested.

Dubois studied the attenuation values of the parenchyma of the cord and the subarachnoid space by serial CT imaging in dogs, and concluded that delayed increase intracavitary opacity might result from slow extracellular distribution of metrizamide, and thus might occur independently of ventricular communication. From lumbar myelography of metrizamide in rabbits, Ekholm et al. suggested that metrizamide passed into the spinal cord by simple diffusion. 


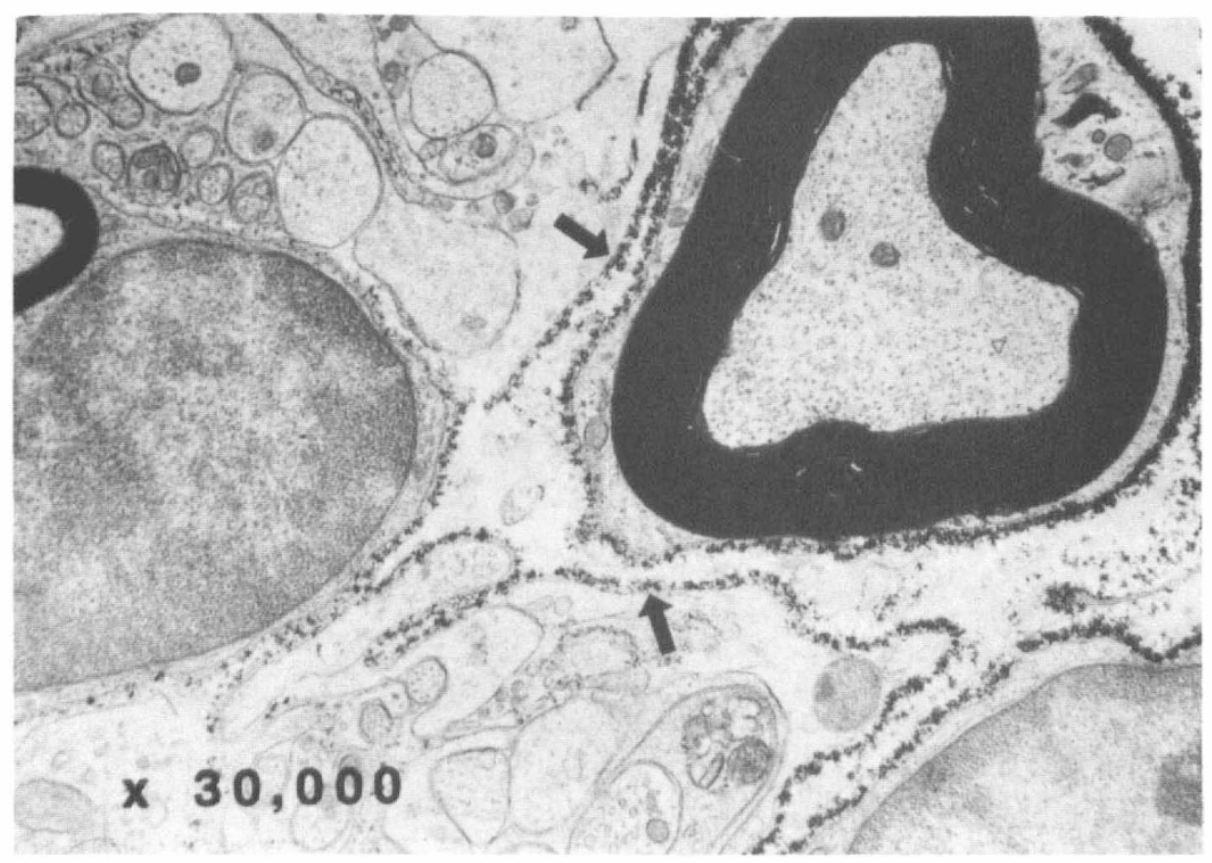

Figure 5 Normal adult rat. Following the subarachnoid perfusion with $\mathrm{LaCl}_{3}$, electron dense lanthanum (arrow) was shown in the extracellular space around the neuropil (Initial magnification $\times 30,000$ ).

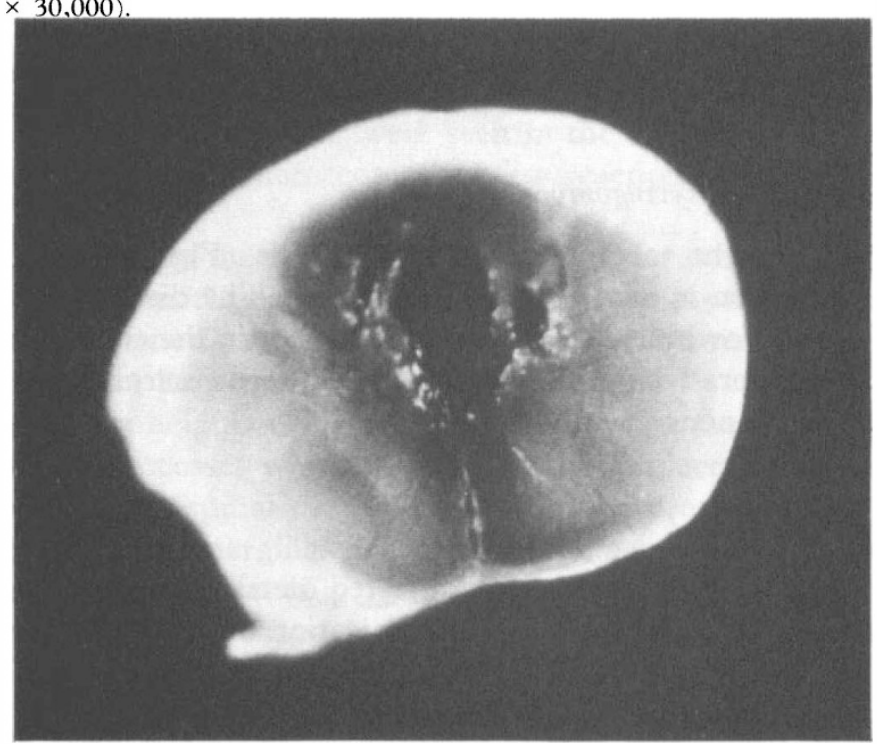

Figure 6 Rat with experimental syringomyelia (8 weeks). It showed syringomyelia and marked adhesion of the arachnoid.

Numerous studies have demonstrated the ready communication between the cerebrospinal fluid and the cerebral extracellular space fluid (e.g., Klatzo, 1964; Brightman, 1964; Levin, 1972; Dunker, 1976). Such studies have largely been confined to the brain. On the other hand, Lee and Olszewski (1960) noted that 


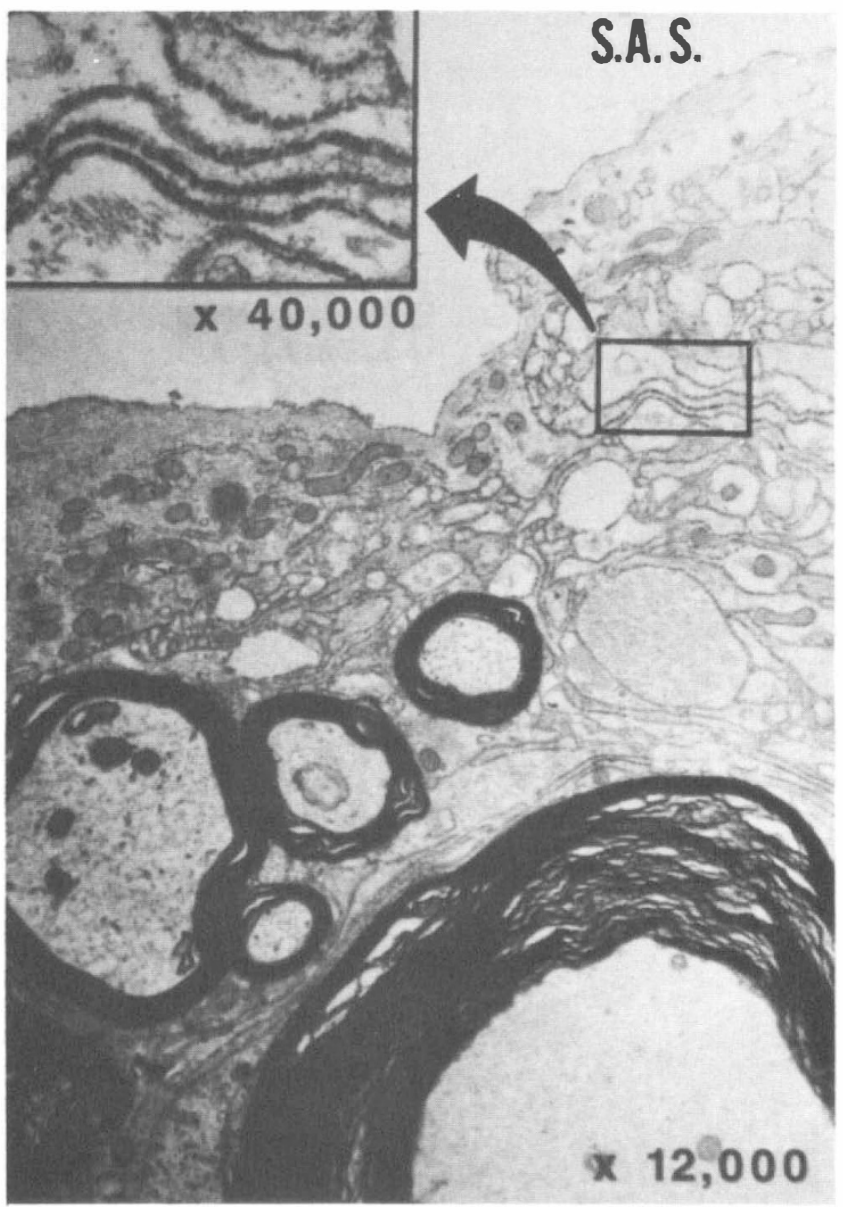

Figure 7 Rat with experimental syringomyelia (6 weeks). Dark HRP reaction products (small square $\times 40,000$ ) were shown in the extracellular space of marginal glia and around the neuropil (Initial magnification $\times 12,000)$.

intrathecally injected radioactive bovine albumin entered the periphery of the spinal cord in cats and dogs. This finding was supported by those of tracer studies with fluorescein labelled serum proteins and a copper-albumin complex (Vogel, 1964; Klatzo, 1964).

We found that ionic lanthanum crossed the marginal glia to enter the parenchyma of both normal cord and syringomyelia of rats. Many deposits of lanthanum were observed in the ECS of the white matter, but none in the ECS of the grey matter. Ionic lanthanum (MW 138.9) is the smallest marker that is visible by electron microscopy (Harned and Owen, 1958). However, lanthanum tends to be lost from tissues during procedures such as fixation, washing, and sectioning. In this study, care was taken to avoid this loss, but it may have occurred. The absence of lanthanum deposits in the ECS of the grey matter does not mean that no lanthanum had diffused to that part. Brightman et al., Turner and Harris and others have shown that HRP is a relatively non-toxic, readily diffusable, cerebral ECS marker protein of 43,000 molecular weight 


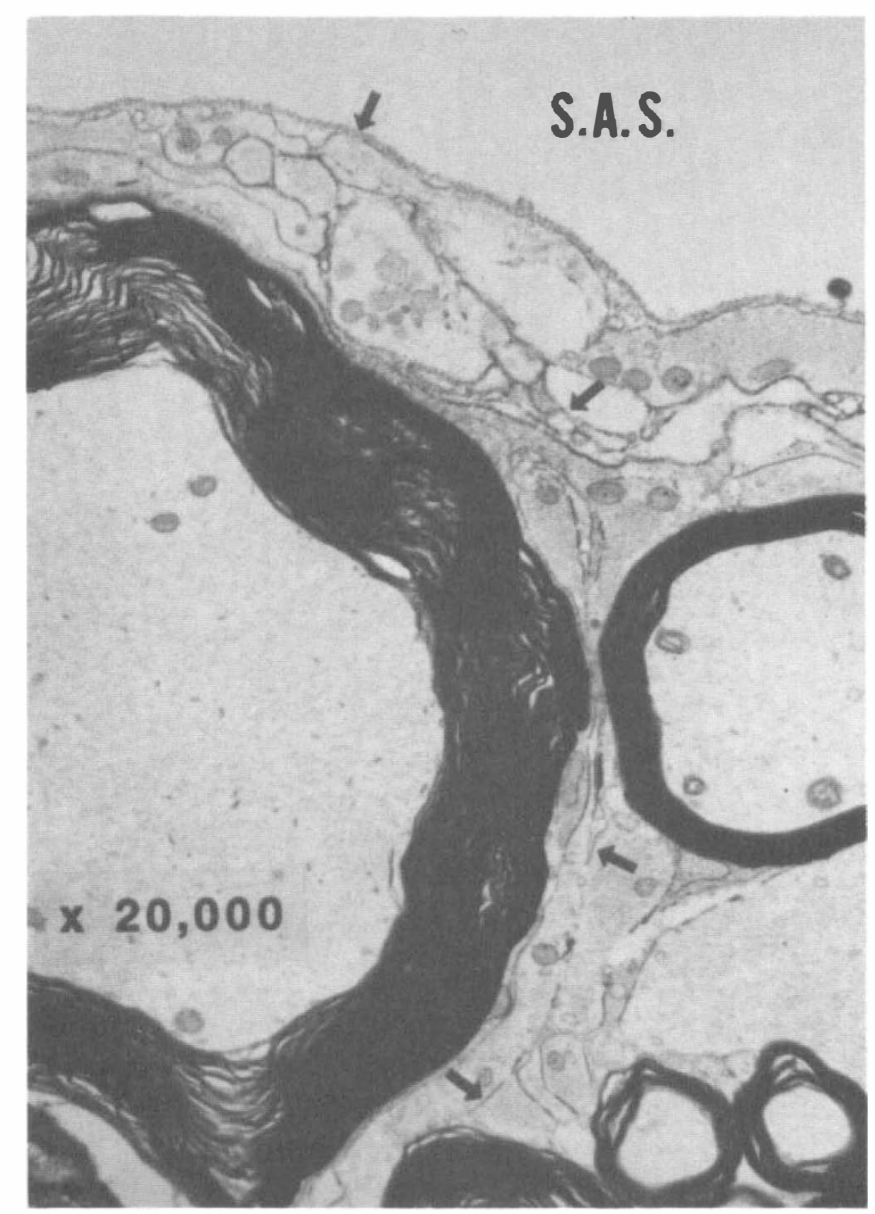

Figure 8 Rat with experimental syringomyelia (6 weeks). Reaction products of HRP migrated deep into the extracellular space of glial cells and the white matter (Initial magnification $x$ 20,000).

with a molecular diameter of 5-6 nm. In normal rats, reactive products of HRP were found in the ECS of the marginal glia, but only a few products of HRP were observed in the ECS of the white matter. In rats with syringomyelia, many reactive products of HRP were observed in the ECS of the white matter.

The ECS of the marginal glia and the neuropil is widely thought to constitute part of a pathway for the movement of small solutes such as fluorescein, Evans Blue and ionic lanthanum, but to be more or less restrictive for large molecular substances such as HRP. The permeability of substances may depend on their molecular size. Conceivably metrizamide ( $M W$ 789) may penetrate into the parenchyma through this pathway. The pathway may grow wider with degeneration of the spinal cord facilitating passage of particles of HRP into the parenchyma. Metrizamide also may pass through this pathway into the parenchyma. 


\section{References}

BoNAFE A 1980 Evaluation of syringomyelia with metrizamide computed tomographic myelography. Fournal of Computer Assisted Tomography 4 (6):797-802.

BRIGHTMAN MW 1965 The distribution within the brain of ferritin injected into cerebrospinal fluid components I: Ependymal distribution. Fournal of Cell Biology 26:99-123.

BRIGHTMAN MW 1965 The distribution within the brain of feritin injected into cerebrospinal fluid components II: Parenchymal distribution. American fournal of Anatomy.

BRIGHTMAN MW, REESE TS 1969 Junctions between intimately apposed cell membranes in the vertebrate brain. Fournal of Cell Biology 40:648-679.

CSERR HR 1974 Relationship between cerebrospinal fluid and interstitial fluid of brain. Federation Proceeding 33:2075-2078.

Drayer BP, Rosenbaum AE 1977 Metrizamide brin penetrance. Acta Radiologica Supplementum 355:280-293.

Dubois PJ, Drayer BP, SAGE M, et al. 1981 Intramedurary penetrance of metrizamide in the dog spinal cord. American fournal of Neuroradiology 2:313-317.

DUNKER RO, HARRIS AB, JENKINS DP 1976 Kinetics of horseradish peroxidase migration through cerebral cortex. Brain Research 118:199-217.

EкhоLM SE, FolEy M, KIDO DK 1984 Lumber myelography with metrizamide in rabbits. Acta Radiologica Diagnosis 25:517-522.

Fenstermacher JD, BradBuRy MWB, DU Boulay G, et al. 1980 The Distribution of ${ }^{125}$ IMetrizamide and ${ }^{125}$ I-diatrizoate between blood, brain, and cerebrospinal fluid in the rabbit. Neuroradiology 19:171-180.

GolMAN K 1973 Distribution and retention of 125I-labeled metrizamide after intravenous and suboccipital injection of rabbit, rat, and cat. Acta Radiologica Supplimentum 335:300-311.

Hashimoto H, Gotow T, IchimuRA T, et al. 1982 Are the arachnoid villi really the main drainage route for the cerebrospinal fluid into the blood stream? An electron microscopic Study. Okajimas Folia Anatomica 58 (4-6):819-836.

IsHerwood I, FAwCITT RA, ForBes W, et al, 1977 Computer tomography of the spinal canal using metrizamide. Acta Radiologica Supplementum 335:299-305.

Klatzo I, Mizuel J, Ferris PJ, et al. 1964 Observations on the passage of the fluorescein labeled serum proteins (FLSP) from the celebrospinal fluid. Fournal of Neuropathology and Experimental Neurology 23:18-35.

LEE JC, OLSZEWSKY J 1960 Penetration of radoactive bovine albumin from cerebrospinal fluid into the brain tissue. Neurology 10:814.

LEviN I, Sisson WB 1972 The Penetration of radiolaveled substances into rabbit brain from subarachnoid space. Brain Research 41:145-153.

MASAKI K, IKATA T, KASHIWAGUCHI S 1987 Clinical experience of iotrolan, a new water-soluble contrast medium, visualization and transmedullary penetration. Seikeigeka 38 (4):577-581.

Michael RS, JohN WC, Evill A, et al. 1982 Brain parenchyma penetration by intrathecal ionic and nonionic contrast media. American fournal of Neuroradiology 3:481-483.

MICHAEL RS 1983 Kinetics of water-soluble contrast media in the central nervous system. American fournal of Radiology 141:815-824.

NABEShima S, REESE TS, LANDIS D, et al. 1975 Junctions in the meninges and marginal glia. fournal of Comparative Neurology 164:127-170.

Nakagawa Y, Cervos NJ, Artigas J 1984 A possible paracellular route for the resolution of hydrocephalic edema. Acta Neuropathologica 64:122-128.

RESJO IM, NASH DCH, FITZ CT 1979 Computed tomographic metrizamide myelography in syringohydromyelia. Radiology 131:405-407.

Shabo Al, MAXWELl DS 1971 The subarachnoid space following introduction of a foreign protein: An electron microscopic study with peroxidase. Fournal of Neuropathology and Experimental Neurology 30:506-524.

VOGEL FS, EvANS JW 1961 Morphologic alterations produced by copper in neural tissues with consideration of the role of the metal in the pathogenesis of Wilson's disease. fournal of Experimental Medicine 113:997-1004.

Westergard E, Deurs B, Bronsted HE 1977 Increased vesicular transfer of horseradish peroxidase across cerebral endthelium, evoked by acute hypertention. Acta Neuropathologica 37:141-152.

WINKLER SS, SACKETT JF 1980 Explanation of metrizamide brain penetration. fournal of Computer Assisted Tomography 4 (2):191-193. 